Article

\title{
Synthesis of Novel Potent Biologically Active $N$-Benzylisatin-Aryl Hydrazones in Comparison with Lung Cancer Drug 'Gefitinib'
}

\author{
Huda S. Al-Salem ${ }^{1, *}$, Hatem A. Abuelizz ${ }^{1}{ }^{\mathbb{D}}$, Iman S. Issa ${ }^{1}$, Amany Z. Mahmoud ${ }^{2,3}{ }^{\mathbb{D}}$, \\ Ali AlHoshani ${ }^{4}$, Md Arifuzzaman ${ }^{5}$ and A. F. M. Motiur Rahman ${ }^{1, * \mathbb{D}}$ \\ 1 Department of Pharmaceutical Chemistry, College of Pharmacy, King Saud University, Riyadh 11451, \\ Saudi Arabia; habuelizz@ksu.edu.sa (H.A.A.); iman_issa69@yahoo.com (I.S.I.) \\ 2 Departments of Pharmaceutics, College of Pharmacy, King Saud University, Riyadh 11451, Saudi Arabia; \\ azmahmoud@ksu.edu.sa \\ 3 Department of Pharmaceutical Medicinal Chemistry, Faculty of Pharmacy, Assiut University, \\ Assiut 71516, Egypt \\ 4 Departments of Pharmacology and Toxicology, College of Pharmacy, King Saud University, Riyadh 11451, \\ Saudi Arabia; ahoshani@ksu.edu.sa \\ 5 College of Pharmacy, Yeungnam University, Gyeongsan 38541, Korea; arifmilon2016@gmail.com \\ * Correspondence: hhalsalem@ksu.edu.sa (H.S.A.-S.); afmrahman@ksu.edu.sa (A.F.M.M.R.); \\ Tel.: +966-11-29-52740 (H.S.A.); +966-11-46-70237 (A.F.M.M.R.)
}

Received: 4 April 2020; Accepted: 13 May 2020; Published: 26 May 2020

Featured Application: Excellent potential scaffold for the development of anticancer therapeutics.

\begin{abstract}
Developing anticancer therapeutics with no/few side effects is a challenge for medicinal chemists. The absence of antibacterial activity of an anticancer drug removes its detrimental effect toward intestinal flora and therefore leads to reduced side effects. Here, a series of novel $\mathrm{N}$-benzylisatin-aryl-hydrazones was designed, synthesized and evaluated for their antimicrobial and antiproliferative activities with SAR and ADME studies, aiming to develop anticancer drugs with no antimicrobial, yet high antiproliferative activities. The results were then compared with the effects of first-line treatments for lung cancer drug Gefitinib. Novel N-benzylisatin-aryl-hydrazones were synthesized from isatin and benzyl bromide in three steps with good to moderate yields. Antimicrobial activity was tested with six Gram-positive/negative bacterial strains, antifungal activity with a fungal strain and antiproliferative activity against 'A549' and 'HeLa cell lines', respectively. As expected, synthesized hydrazones reveled no effects on any of the strains of bacteria and fungi up to $100-\mu \mathrm{g} /$ disc concentration. However, four compounds showed two-to-four fold antiproliferative activity over Gefitinib. For instance, $\mathrm{IC}_{50}$ of a compound $(\mathbf{6 c})$ shows concentration of $4.35 \mu \mathrm{M}$, whereas gefitinib shows $15.23 \mu \mathrm{M}$ against 'A549.' ADME predicted studies reveled that our synthesized hydrazones exhibited higher ADME values than the Gefitinib. Therefore, our synthesized hydrazones can be an excellent scaffold for the development of anticancer therapeutics after considering further investigations.
\end{abstract}

Keywords: N-benzylisatin-aryl hydrazones; gefitinib; cancer; A549 cell lines

\section{Introduction}

Cancer is a life-threatening disease; one of the major challenges for relieving its burden is to develop highly effective drugs with specificity for cancer, but few to no side effects on normal mammalian 
cells [1]. Gefitinib, the epidermal growth factor receptor (EGFR) inhibitor, was added recently to the Food and Drug Administration (FDA) list of the recommended first-line treatments for lung cancer [2]. Gefitinib is particularly recommended for non-small cell lung cancer (NSCLC) type [3]. Although that erlotinib and afatinib are also included in this list, gefitinib may be the most tolerable [4]. The most common adverse effects of this group of drugs were gastrointestinal diarrhea, nausea and/or vomiting which is due to the toxic effect of the drug on normal flora $[5,6]$. The absence of antibacterial activity of the anticancer drug removes its detrimental effect against intestinal flora, suggesting a highly promising new strategy for the development of anticancer drugs with reduced side effects [7]. In 2008, O'Shea and Moser reported [8] that molecular weight is an important factor for antimicrobial activity. They did experiment on 147 antibacterial and 4623 non-antibacterial compounds and found average molecular weight for an antibacterial compound were $812 \mathrm{Da}$ (Gram ' ${ }^{\prime}$ ' positive) and $414 \mathrm{Da}$ (Gram ' - ' negative). In addition, another important factor for becoming an antimicrobially active compound is polarity. According to the Lipinski's rule of five, also known as Pfizer's rule of five or simply the rule of five (RO5) [9], 70.4\% of the antibacterial active compounds showed $\log \mathrm{P}$ values ranges from $0-5$. Therefore, in order to target a compound with no/little antimicrobial activity, lower molecular weight and with more $\log \mathrm{P}$ values should be taken into consideration, isatin and its derivatives with low molecular weight could be a choice of interests [10-12]. Isatin, a natural compound, is known for more than a century and still being used extensively in medicinal compound synthesis [13-19]. It has been reported that, various substituents on isatin nucleus displayed numerous biological activities [12,20]. In recent years, number of isatin derivatives were reported with extensive biological activities [21-25], included EGFR activity [26]. N-benzylisatin hydrazones of fluorescein had showed antiproliferative activity as well as topo II inhibitory activity [27]. Moreover, two series of hydrazone derivatives has been reported recently with antiproliferative activity $[28,29]$. A number of marketed drugs and potential anticancer agents having isatin moiety, depicted in Figure 1, inspired us to synthesize a series of isatin hydrazones having $N$-benzyl protection at 1-position of isatin and hydrazone formation at 3-position with various aryl substituents. We thus anticipating that simplification of isatin molecule would prevent its inhibitory effect on different microbes, providing more selectivity in the action on cancerous cell lines with less/no toxicity on the gastrointestinal tract (GIT) lining cells. Considering the above points and the importance of the development of anticancer therapeutics with no/few side effects, we therefore designed and synthesized a series of $N$-protected $N$-benzylisatin-aryl hydrazones with a lower molecular weight (353-418 Da), evaluated their antibacterial activity against two Gram-positive, four Gram-negative bacterial strains and antifungal activity against Candida albicans NRRL Y-477, and antiproliferative activities against non-small cell lung cancer cell lines 'A549', as well as human cervical cancer cells lines 'HeLa.' In addition, for comparing the potency of the synthesized compounds, "gefitinib" was used as a positive control for antiproliferative activity evaluation.

In order to consider a compound as drug molecule, it is necessary to test their drug likeness properties as well as the analysis of physiological descriptors such as absorption, distribution, metabolism and excretion (ADME). ADME are important physiological descriptors of chemical compounds for selecting highly potential drug targets. However, testing a wide range of compounds directly in clinical or pre-clinical phase is extensively time consuming and costly. Moreover, ADME was considered as the last step of drug development where many drugs (approximately 60\%) were failed after all the procedures. To solve these problems, recent experiments utilizes in silico ADME tools as the first step to shorten the amount of target compounds, by calculating predicted ADME properties and by discarding the compounds with unsatisfactory ADME values from the drug designing pipe line [30]. This prediction enabled us to identify potent drug candidate by analyzing the properties of the designed compounds. Therefore, we studied the ADME predicted parameters of the synthesized compounds (6a-j) using in silico ADME tools and compared them with that of "gefitinib". 


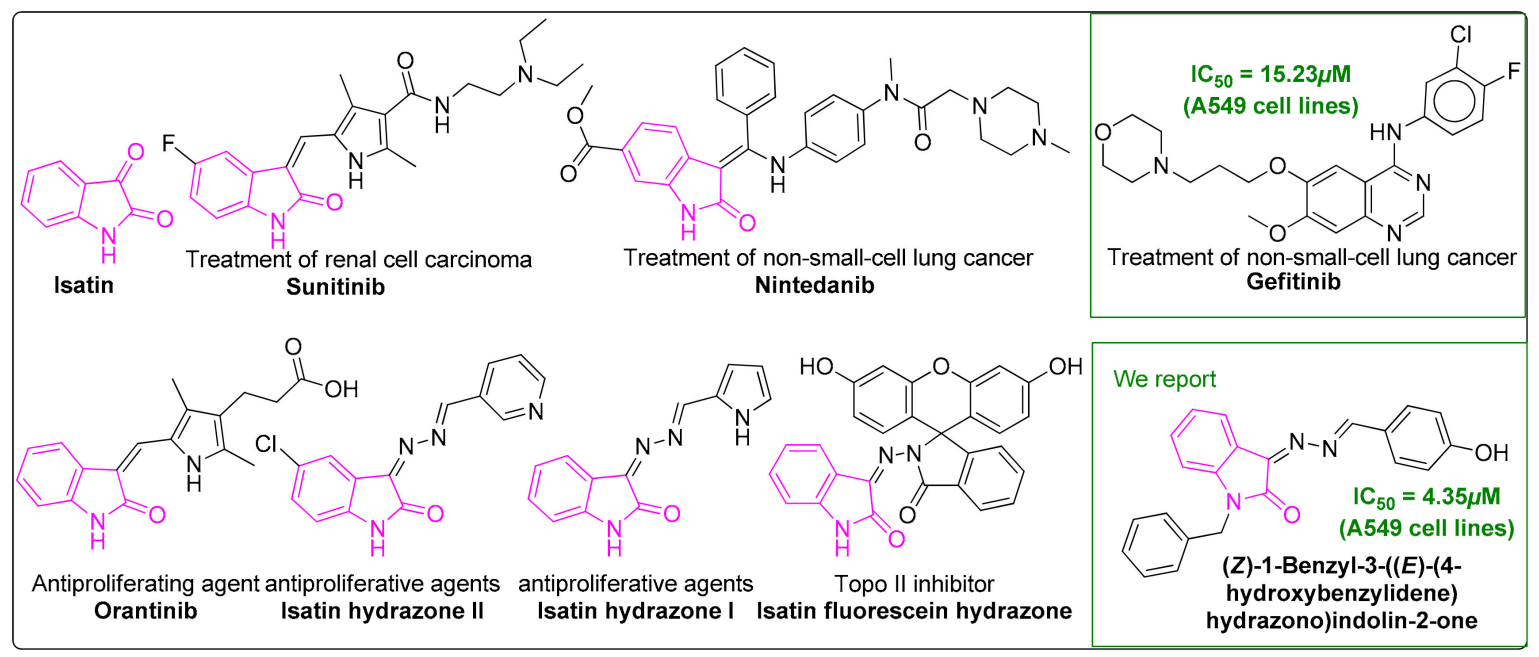

Figure 1. Isatin moiety containing active \& potential drugs and gefitinib.

\section{Materials and Methods}

2.1. Chemicals and Solvents Were of Commercial Reagent Grade (Sigma-Aldrich, St. Louis, MO, USA) and Used without Further Purification

The progress of reactions and purity of reactants and products were checked using pre-coated silica gel 60 aluminum TLC sheets with fluorescent indicator UV254 of Macherey-Nagel, and detection was carried out with ultraviolet light $(254 \mathrm{~nm})$. Melting points were determined on a Fisher ScientificTM digital melting point apparatus (model number IA9100) and are uncorrected. Electrospray ionization (ESI) mass spectrometry (MS) experiments were performed using an Agilent high performance liquid chromatography (HPLC) 1200 connected to an Agilent 6320 ion trap mass spectrometer fitted with an electrospray ionization (ESI) ion source (Agilent Technologies, Palo Alto, CA, USA). Infrared spectra were recorded as $\mathrm{KBr}$ disks using the Fourier Transform Infrared Spectrophotometer of Shimadzu; model: IR affinity-1S (Shimadzu, Tokyo, Japan). NMR spectra were taken on Agilent-NMR-VNMRS $600 \mathrm{MHz}$ spectrometer (Agilent Technologies, Palo Alto, CA, USA) and DMSO-d6 was used as solvent.

\subsection{Preparation and Characterization of Target Compounds}

\subsubsection{1-Benzylindoline-2,3-dione (3)}

Orange powder $(95 \%) \mathrm{mp}=130-131^{\circ} \mathrm{C}\left(\right.$ Lit. [31] $\left.\mathrm{mp} .=125-126^{\circ} \mathrm{C}\right)$.

\subsection{2. (Z)-1-Benzyl-3-hydrazonoindolin-2-one (4)}

Yellow powder $(90 \%) \mathrm{mp}=124.5-126^{\circ} \mathrm{C}\left(\right.$ Lit. $\left.[32,33] \mathrm{mp} .=125-126^{\circ} \mathrm{C}\right) . \mathrm{ESI} \mathrm{mass} \mathrm{m} / \mathrm{z}=252$ $[\mathrm{M}+\mathrm{H}]^{+}$.

\subsubsection{General Procedure for the Synthesis of $\mathbf{6 a} \mathbf{a}-\mathbf{j}$}

To a mixture of isatin monohydrazone $(1 \mathrm{mmol})$ and substituted aryl aldehyde $(1 \mathrm{mmol})$ in $10 \mathrm{~mL}$ ethanol, a few drops of glacial acetic acid was added. The reaction mixture was refluxed for $4 \mathrm{~h}$. The precipitate solid was filtered, washed with cold ethanol and air dried to obtain the target compounds $(\mathbf{6 a}-\mathbf{j})$, which was then further purified by recrystallization using methanol. ${ }^{13} \mathrm{CNMR}$ spectra for $\mathbf{6} \mathbf{a}-\mathbf{j}$ is available in the Supplementary Materials file.

1-Benzyl-3-((4-(dimethylamino) benzylidene)hydrazono)indolin-2-one (6a)

Dark red solid $(53 \%) \mathrm{mp}=183-184^{\circ} \mathrm{C}$. IR $(\mathrm{KBr}): v_{\max }\left(\mathrm{cm}^{-1}\right): 2926(\mathrm{C}-\mathrm{H}$ aliphatic $), 1707(\mathrm{C}=\mathrm{O})$, $1606(\mathrm{C}=\mathrm{N}), 1558(\mathrm{C}=\mathrm{N}) .{ }^{1} \mathrm{H}$ NMR (DMSO-d6, $600 \mathrm{MHz}: \delta 3.06$ (s, 6H, N(CH$\left.)_{2}\right), 4.97\left(\mathrm{~s}, 2 \mathrm{H}, \mathrm{N}-\mathrm{CH}_{2}-\mathrm{Ph}\right)$, 
$6.84(\mathrm{~d}, J=7.8,2 \mathrm{H}, \operatorname{ArH}), 6.98(\mathrm{~d}, J=7.2,1 \mathrm{H}, \operatorname{ArH}), 7.15(\mathrm{t}, 1 \mathrm{H}, \operatorname{ArH}), 7.26(\mathrm{~s}, 1 \mathrm{H}, \operatorname{ArH}), 7.34(\mathrm{~m}$, $5 \mathrm{H}, \mathrm{ArH}), 7.85(\mathrm{~d}, J=7.8,2 \mathrm{H}, \mathrm{ArH}), 8.30(\mathrm{~d}, J=7.2,1 \mathrm{H}, \mathrm{ArH}), 8.67(\mathrm{~s}, 1 \mathrm{H}, \mathrm{N}=\mathrm{CH}) \mathrm{ppm} .{ }^{13} \mathrm{C}$ NMR (DMSO-d6, 150 MHz: $\delta$ 166.41, 164.43, 153.76, 149.56, 144.80, 136.70, 133.09, 131.97, 129.18, 129.08, $127.99,127.68,123.35,120.76,117.04,112.30,110.22,43.11$ and $40.14 \mathrm{ppm}$. ESI mass $\mathrm{m} / \mathrm{z}=383[\mathrm{M}+\mathrm{H}]^{+}$.

1-Benzyl-3-(((4-chlorocyclohexa ${ }^{-1}$, 5-dien-1-yl) methylene)hydrazono)indolin-2-one (6b)

Dark red solid $(30 \%) \mathrm{mp}=210-211^{\circ} \mathrm{C}$. IR $(\mathrm{KBr}): v_{\max }\left(\mathrm{cm}^{-1}\right): 2918$ (C-H aliphatic), $1718(\mathrm{C}=\mathrm{O})$, $1591(\mathrm{C}=\mathrm{N}) .{ }^{1} \mathrm{H}$ NMR (DMSO-d6, $600 \mathrm{MHz}: \delta 4.97\left(\mathrm{~s}, 2 \mathrm{H}, \mathrm{N}-\mathrm{CH}_{2}-\mathrm{Ph}\right), 7.00(\mathrm{~d}, J=7.8,1 \mathrm{H}, \mathrm{ArH}), 7.08(\mathrm{t}$, $1 \mathrm{H}, \mathrm{ArH}), 7.24-7.44(\mathrm{~m}, 6 \mathrm{H}, \mathrm{ArH}), 7.64(\mathrm{~d}, J=7.8,2 \mathrm{H}, \mathrm{ArH}), 7.92(\mathrm{~d}, J=6.6,1 \mathrm{H}, \mathrm{ArH}), 8.05(\mathrm{~d}, J=7.8$, $2 \mathrm{H}, \mathrm{ArH}), 8.66\left(\mathrm{~s}, 1 \mathrm{H}, \mathrm{N}=\mathrm{CH}\right.$ ) ppm. ${ }^{13} \mathrm{C}$ NMR (DMSO-d6, $150 \mathrm{MHz}: \delta 163.69,159.86,150.00,145.57$, 137.31, 136.45, 134.18, 132.66, 131.03, 129.87, 129.20, 128.00, 127.72, 123.60, 116.30, 110.63 and 43.23 ppm. ESI mass $\mathrm{m} / \mathrm{z}=374\left[\mathrm{M}\left({ }^{35} \mathrm{Cl}\right)+\mathrm{H}\right]^{+}, 376\left[\mathrm{M}\left({ }^{37} \mathrm{Cl}\right)+\mathrm{H}\right]^{+}$.

1-Benzyl-3-((4-hydroxybenzylidene)hydrazono)indolin-2-one (6c)

Orange solid $(50 \%) \mathrm{mp}=235-236^{\circ} \mathrm{C}$. IR $(\mathrm{KBr}): v_{\max }\left(\mathrm{cm}^{-1}\right): 3329(\mathrm{O}-\mathrm{H}), 3022$ (C-H aliphatic), $1705(\mathrm{C}=\mathrm{O}), 1608(\mathrm{C}=\mathrm{N}), 1589(\mathrm{C}=\mathrm{N}) .{ }^{1} \mathrm{H}$ NMR (DMSO-d6, $600 \mathrm{MHz}: \delta 4.97\left(\mathrm{~s}, 2 \mathrm{H}, \mathrm{N}-\mathrm{CH}_{2}-\mathrm{Ph}\right), 6.92(\mathrm{~d}$, $J=7.8,2 \mathrm{H}, \mathrm{ArH}), 6.98(\mathrm{~d}, J=7.2,1 \mathrm{H}, \mathrm{ArH}), 7.08(\mathrm{t}, 1 \mathrm{H}, \mathrm{ArH}), 7.22-7.4(\mathrm{~m}, 6 \mathrm{H}, \mathrm{ArH}), 7.73(\mathrm{bs}, 1 \mathrm{H}, \mathrm{OH})$, $7.86(\mathrm{~d}, J=7.2,2 \mathrm{H}, \mathrm{ArH}), 8.15(\mathrm{~d}, J=7.8,1 \mathrm{H}, \mathrm{ArH}), 8.65(\mathrm{~s}, 1 \mathrm{H}, \mathrm{N}=\mathrm{CH})$ ppm. ${ }^{13} \mathrm{C}$ NMR (DMSO-d6, 150 MHz: $\delta 164.32,164.11,162.32,150.79,150.31,145.17,136.56,133.62,132.08,129.29,129.18,127.96$, $127.49,124.88,123.49$ and $43.14 \mathrm{ppm}$. ESI mass $\mathrm{m} / \mathrm{z}=356[\mathrm{M}+\mathrm{H}]^{+}$.

1-Benzyl-3-((2-bromobenzylidene)hydrazono)indolin-2-one (6d)

Orange solid $(65 \%) \mathrm{mp}=173-174{ }^{\circ} \mathrm{C}$. IR $(\mathrm{KBr}): v_{\max }\left(\mathrm{cm}^{-1}\right): 2935(\mathrm{C}-\mathrm{H}$ aliphatic), $1722(\mathrm{C}=\mathrm{O})$, 1606 (C=N). ${ }^{1} \mathrm{H}$ NMR (DMSO-d6, $600 \mathrm{MHz}: \delta 4.95$ (s, 2H, N-CH $\left.2-\mathrm{Ph}\right), 6.99(\mathrm{~d}, J=4.8,1 \mathrm{H}, \mathrm{ArH}), 7.06$ (t, 1H, ArH), $7.25(\mathrm{~m}, 1 \mathrm{H}, \mathrm{ArH}), 7.3-7.37(\mathrm{~m}, 4 \mathrm{H}, \mathrm{ArH}), 7.4(\mathrm{t}, 1 \mathrm{H}, \mathrm{ArH}), 7.51(\mathrm{t}, 1 \mathrm{H}, \mathrm{ArH}), 7.58(\mathrm{t}, 1 \mathrm{H}$, ArH), $7.78(\mathrm{~d}, J=8.4,1 \mathrm{H}, \mathrm{ArH}), 7.86(\mathrm{~d}, J=7.2,1 \mathrm{H}, \mathrm{ArH}), 8.22(\mathrm{~d}, J=7.2,1 \mathrm{H}, \mathrm{ArH}), 8.75(\mathrm{~s}, 1 \mathrm{H}, \mathrm{N}=\mathrm{CH})$ ppm. ${ }^{13}$ C NMR (DMSO-d6, 150 MHz: $\delta$ 163.57, 158.38, 149.98, 145.68, 136.37, 134.37, 134.28, 134.15, $132.16,129.31,129.19,129.15,129.10,128.00,127.71,125.68,123.64,116.16,116.16,110.68$ and $43.22 \mathrm{ppm}$. ESI mass $\mathrm{m} / \mathrm{z}=418\left[\mathrm{M}\left({ }^{79} \mathrm{Br}\right)+\mathrm{H}\right]^{+}, 420\left[\mathrm{M}\left({ }^{81} \mathrm{Br}\right)+\mathrm{H}\right]^{+}$.

1-Benzyl-3-((E)-(3-bromobenzylidene)hydrazono)indolin-2-one (6e)

Orange solid (52\%) $\mathrm{mp}=173-174{ }^{\circ} \mathrm{C}$. IR (KBr): $v_{\max }\left(\mathrm{cm}^{-1}\right)$ : 2920 (C-H aliphatic), $1720(\mathrm{C}=\mathrm{O})$, $1624(\mathrm{C}=\mathrm{N}), 1573(\mathrm{C}=\mathrm{N}) .{ }^{1} \mathrm{H}$ NMR (DMSO-d6, $600 \mathrm{MHz}: \delta 4.97\left(\mathrm{~s}, 2 \mathrm{H}, \mathrm{N}-\mathrm{CH}_{2}-\mathrm{Ph}\right), 7.01(\mathrm{~d}, J=8.4,1 \mathrm{H}$, ArH), $7.09(\mathrm{t}, 1 \mathrm{H}, \mathrm{ArH}), 7.25(\mathrm{~m}, 1 \mathrm{H}, \mathrm{ArH}), 7.24-7.38(\mathrm{~m}, 4 \mathrm{H}, \mathrm{ArH}), 7.41(\mathrm{t}, 1 \mathrm{H}, \mathrm{ArH}), 7.54(\mathrm{t}, 1 \mathrm{H}, \mathrm{ArH})$, $7.79(\mathrm{~d}, J=8.4,1 \mathrm{H}, \mathrm{ArH}), 7.86(\mathrm{~d}, J=7.8,1 \mathrm{H}, \mathrm{ArH}), 8.02(\mathrm{~d}, J=7.8,1 \mathrm{H}, \mathrm{ArH}), 8.12(\mathrm{~s}, 1 \mathrm{H}, \mathrm{ArH}), 8.60$ (s, $1 \mathrm{H}, \mathrm{N}=\mathrm{CH}$ ) ppm. ${ }^{13} \mathrm{C}$ NMR (DMSO-d6, $150 \mathrm{MHz}: \delta 163.60,158.67,149.59,145.61,136.43,136.10$, 135.22, 134.12, 132.07, 131.88, 129.21, 129.10, 128.01, 127.73, 127.66, 123.65, 122.86, 116.20, 110.67 and $43.25 \mathrm{ppm}$. ESI mass $\mathrm{m} / \mathrm{z}=418\left[\mathrm{M}\left({ }^{79} \mathrm{Br}\right)+\mathrm{H}\right]^{+}, 420\left[\mathrm{M}\left({ }^{81} \mathrm{Br}\right)+\mathrm{H}\right]^{+}$.

1-Benzyl-3-((4-bromobenzylidene)hydrazono)indolin-2-one (6f)

Orange solid $(68 \%) \mathrm{mp}=216-217^{\circ} \mathrm{C}$. IR $(\mathrm{KBr}): v_{\max }\left(\mathrm{cm}^{-1}\right): 2922(\mathrm{C}-\mathrm{H}$ aliphatic), $1720(\mathrm{C}=\mathrm{O})$, $1602(\mathrm{C}=\mathrm{N}) .{ }^{1} \mathrm{H}$ NMR (DMSO-d6, $600 \mathrm{MHz}: \delta 4.95$ (s, 2H, N-CH $\left.-\mathrm{Ph}\right), 6.85$ (d, J = 7.2, 1H, ArH), 7.05 $(\mathrm{t}, 1 \mathrm{H}, \mathrm{ArH}), 7.22-7.42(\mathrm{~m}, 6 \mathrm{H}, \mathrm{ArH}), 7.76(\mathrm{~d}, J=7.8,2 \mathrm{H}, \mathrm{ArH}), 7.9(\mathrm{~d}, J=7.8,3 \mathrm{H}, \mathrm{ArH}), 8.60(\mathrm{~s}, 1 \mathrm{H}$, $\mathrm{N}=\mathrm{CH}$ ) ppm. ${ }^{13} \mathrm{C}$ NMR (DMSO-d6, $150 \mathrm{MHz}: \delta 163.67,159.89,149.91,145.53,136.42,134.18,132.94$, $132.77,131.15,129.19,128.01,127.70,126.34,123.60,116.26,110.61$ and $43.20 \mathrm{ppm}$. ESI mass $\mathrm{m} / \mathrm{z}=418$ $\left[\mathrm{M}\left({ }^{79} \mathrm{Br}\right)+\mathrm{H}\right]^{+}, 420\left[\mathrm{M}\left({ }^{81} \mathrm{Br}\right)+\mathrm{H}\right]^{+}$. 
1-Benzyl-3-((2-methyl benzylidene)hydrazono)indolin-2-one (6g)

Orange solid $(60 \%) \mathrm{mp}=176-177^{\circ} \mathrm{C}$. IR $(\mathrm{KBr}): v_{\max }\left(\mathrm{cm}^{-1}\right): 2920$ (C-H aliphatic), $1716(\mathrm{C}=\mathrm{O})$, $1624(\mathrm{C}=\mathrm{N}) .{ }^{1} \mathrm{H}$ NMR (DMSO-d6, $600 \mathrm{MHz}: \delta 2.53\left(\mathrm{t}, 3 \mathrm{H}, \mathrm{Ph}-\mathrm{CH}_{3}\right), 4.96\left(\mathrm{~s}, 2 \mathrm{H}, \mathrm{N}-\mathrm{CH}_{2}-\mathrm{Ph}\right), 7.0(\mathrm{~d}$, $J=7.8,1 \mathrm{H}, \mathrm{ArH}), 7.07(\mathrm{t}, 1 \mathrm{H}, \mathrm{ArH}), 7.23-7.41(\mathrm{~m}, 8 \mathrm{H}, \mathrm{ArH}), 7.45(\mathrm{t}, 1 \mathrm{H}, \mathrm{ArH}), 7.95(\mathrm{~d}, J=7.8,1 \mathrm{H}, \mathrm{ArH})$, $8.08(\mathrm{~d}, J=7.2,1 \mathrm{H}, \mathrm{ArH}), 8.85(\mathrm{~s}, 1 \mathrm{H}, \mathrm{N}=\mathrm{CH}) \mathrm{ppm} .{ }^{13} \mathrm{C}$ NMR (DMSO-d6, $150 \mathrm{MHz}: \delta 163.80,159.92$, 149.81, 145.45, 139.86, 136.46, 134.04, 132.44, 131.80, 131.72, 129.19, 128.91, 128.07, 128.00, 127.71, 127.09, $123.54,116.33,110.59,43.19$ and 19.59 ppm. ESI mass $\mathrm{m} / \mathrm{z}=354[\mathrm{M}+\mathrm{H}]^{+}$.

1-Benzyl-3-((3-methylbenzylidene)hydrazono)indolin-2-one (6h)

Pale orange solid $(60 \%) \mathrm{mp}=138-139^{\circ} \mathrm{C}$. IR $(\mathrm{KBr}): v_{\max }\left(\mathrm{cm}^{-1}\right): 2920$ (C-H aliphatic), $1718(\mathrm{C}=\mathrm{O})$, $1670(\mathrm{C}=\mathrm{N}), 1606(\mathrm{C}=\mathrm{N}) .{ }^{1} \mathrm{H}$ NMR (DMSO-d6, $600 \mathrm{MHz}: \delta 2.38\left(\mathrm{~s}, 3 \mathrm{H}, \mathrm{Ph}_{-} \mathrm{CH}_{3}\right), 4.95\left(\mathrm{~s}, 2 \mathrm{H}, \mathrm{N}-\mathrm{CH}_{2}-\mathrm{Ph}\right)$, $7.0(\mathrm{~d}, J=8.4,1 \mathrm{H}, \mathrm{ArH}), 7.07(\mathrm{t}, 1 \mathrm{H}, \mathrm{ArH}), 7.22-7.42(\mathrm{~m}, 7 \mathrm{H}, \mathrm{ArH}), 7.45(\mathrm{t}, 1 \mathrm{H}, \mathrm{ArH}), 7.76(\mathrm{~s}, 1 \mathrm{H}, \mathrm{ArH})$, $7.78(\mathrm{~d}, J=7.8,1 \mathrm{H}, \mathrm{ArH}), 7.95(\mathrm{~d}, J=7.2,1 \mathrm{H}, \mathrm{ArH}), 8.85(\mathrm{~s}, 1 \mathrm{H}, \mathrm{N}=\mathrm{CH}) \mathrm{ppm} .{ }^{13} \mathrm{C}$ NMR (DMSO-d6, 150 MHz: $\delta$ 163.74, 161.14, 149.83, 145.45, 139.06, 136.46, 134.03, 133.75, 133.43, 129.98, 129.62, 129.19, $129.13,129.10,127.99,127.71,126.45,123.60,117.81,116.34,110.57,43.19$ and 21.35 ppm. ESI mass m/z $=354[\mathrm{M}+\mathrm{H}]^{+}$.

1-Benzyl-3-((4-methylbenzylidene)hydrazono)indolin-2-one (6i)

Pale red solid $(82 \%) \mathrm{mp}=191-192{ }^{\circ} \mathrm{C}$. IR (KBr): $v_{\max }\left(\mathrm{cm}^{-1}\right): 2920$ (C-H aliphatic), $1722(\mathrm{C}=\mathrm{O})$, $1683(\mathrm{C}=\mathrm{N}), 1602(\mathrm{C}=\mathrm{N}) .{ }^{1} \mathrm{H}$ NMR (DMSO-d6, $600 \mathrm{MHz}: \delta 2.38$ (s, 3H, Ph-CH $), 4.95$ (s, 2H, N-CH $-\mathrm{Ph}$ ), $6.99(\mathrm{~d}, J=7.8,1 \mathrm{H}, \mathrm{ArH}), 7.07(\mathrm{t}, 1 \mathrm{H}, \mathrm{ArH}), 7.22-7.41(\mathrm{~m}, 8 \mathrm{H}, \mathrm{ArH}), 7.87(\mathrm{~d}, J=8.4,2 \mathrm{H}, \mathrm{ArH}), 8.00(\mathrm{~d}$, $J=7.2,1 \mathrm{H}, \mathrm{ArH}$ ), 8.65 (s, 1H, N=CH) ppm. ${ }^{13} \mathrm{C}$ NMR (DMSO-d6, $150 \mathrm{MHz}: \delta 163.83,161.88,150.09$, $145.40,143.17,136.48,133.96,131.14,130.33,129.53,129.19,127.98,127.70,123.55,116.43,110.54,43.18$ and $21.74 \mathrm{ppm}$. ESI mass $\mathrm{m} / \mathrm{z}=354[\mathrm{M}+\mathrm{H}]^{+}$.

1-Benzyl-3-((4-(methylthio)benzylidene)hydrazono)indolin-2-one (6j)

Orange solid $(65 \%) \mathrm{mp}=177-178^{\circ} \mathrm{C}$. IR $(\mathrm{KBr}): v_{\max }\left(\mathrm{cm}^{-1}\right): 2926$ (C-H aliphatic), $1720(\mathrm{C}=\mathrm{O})$, $1606(\mathrm{C}=\mathrm{N}) .{ }^{1} \mathrm{H}$ NMR (DMSO-d6, $600 \mathrm{MHz}: \delta 2.49\left(\mathrm{~s}, 3 \mathrm{H}, \mathrm{S}-\mathrm{CH}_{3}\right), 4.97\left(\mathrm{~s}, 2 \mathrm{H}, \mathrm{N}_{-} \mathrm{CH}_{2}-\mathrm{Ph}\right), 7.01(\mathrm{~d}$, $J=7.8,1 \mathrm{H}, \mathrm{ArH}), 7.09(\mathrm{t}, 1 \mathrm{H}, \mathrm{ArH}), 7.27(\mathrm{~m}, 1 \mathrm{H}, \mathrm{ArH}) 7.3-7.42(\mathrm{~m}, 5 \mathrm{H}, \mathrm{ArH}), 7.43(\mathrm{~d}, J=8.4,2 \mathrm{H}, \mathrm{ArH})$, $7.92(\mathrm{~d}, J=7.8,2 \mathrm{H}, \mathrm{ArH}), 8.04(\mathrm{~d}, J=7.2,1 \mathrm{H}, \mathrm{ArH}), 8.70(\mathrm{~s}, 1 \mathrm{H}, \mathrm{N}=\mathrm{CH}) \mathrm{ppm} .{ }^{13} \mathrm{C}$ NMR (DMSO-d6, 150 MHz: $\delta 163.89,162.07,150.20,145.41,144.96,136.51,133.95,130.01,129.88,129.20,127.99,127.72$, $126.07,123.55,116.50,110.54,43.20$ and $14.50 \mathrm{ppm}$. ESI mass $\mathrm{m} / \mathrm{z}=386[\mathrm{M}+\mathrm{H}]^{+}$.

\subsection{Antimicrobial Evaluation}

Different organisms were selected to cover the Gram-positive and Gram-negative bacteria; namely Staphylococcus aureus ATCC 29213 and Bacillus subtilis ATCC 3366 as Gram-positive, Escherichia coli ATCC 25922, Klebsiella pneumoniae ATCC 13883, Salmonella typhi ATCC 25566 and Pseudomonas aeruginosa ATCC 27853 as Gram-negative microorganisms. Antifungal effect was investigated using Candida albicans NRRL Y-477. Disc diffusion method was used as the method of the microbiological study, where the standard antibiotic discs were Ampicillin $10 \mu \mathrm{g} / \mathrm{disc}$, erythromycin $10 \mu \mathrm{g} / \mathrm{disc}$ and anti-fungal fluconazole. The negative control was DMSO (100\%). The result was collected to estimate the existence of antibacterial efficiencies of the prepared compounds depending on the criterion procedure by Bauer et al [34]. Bacterial culture, at first, which is adjusted to $0.5 \mathrm{McFarland}$ standards, was applied to Mueller Hinton agar plates in-house. After that, the plates were dried for $15 \mathrm{~min}$. and then applied for the sensitivity test. The discs were impregnated with the tested compounds on the $\mathrm{MH}$ agar surface together with the control antibiotic and the negative control and incubated at $37^{\circ} \mathrm{C}$ for $24 \mathrm{~h}$. After incubation, the plates were examined for any inhibition zone. To ensure reliability of the result, the test was repeated three times. 


\subsection{Antiproliferative Assay}

A549 and Hela cells (Produced from ATCC, Rockville, MD, USA) were cultured in DMEM (Dulbecco's Modified Eagle Medium), supplemented with $10 \% \mathrm{FBS}, 100 \mathrm{mg} / \mathrm{mL}$ of streptomycin and $100 \mathrm{U} / \mathrm{mL}$ of penicillin at $37^{\circ} \mathrm{C}$ in a $5 \%-\mathrm{CO}_{2}$ humidified atmosphere. Cell cytotoxicity assay was done by MTT method [35]; 3-(4,5-dimethylthiazol-2-yl)-2,5-diphenyltetrazolium bromide method. Concisely, cells were seeded in 96 -well tissue culture plates and incubated for $48 \mathrm{~h}$ at $37^{\circ} \mathrm{C}, 5 \% \mathrm{CO}_{2}$. The culture medium was removed and replaced with fresh medium containing the synthesized compounds in different concentrations to the wells and incubated for another $24 \mathrm{~h}$. Thereafter, the MTT solution $(1 \mathrm{mg} / \mathrm{mL})$ was added and incubated for $3 \mathrm{~h}$. To dissolve the reduced MTT crystals, one hundred microliters of ethanol was added to each well, then, the optical density of each well was measured at 492/630 nm with enzyme immunoassay instrument.

\subsection{In Silico ADME Prediction Analysis}

In this experiment, in silico ADME analysis was done in QikProp module of Schrodinger Maestro [36] where the important physiological descriptors like predicted $\mathrm{IC}_{50}$ for blocking hERG K+ channel in vitro, predicted octanol or water partition coefficient $[\log \mathrm{P}(\mathrm{o} / \mathrm{w})]$, number of hydrogen bond acceptors (HBA), number of hydrogen bond donors (HBD), predicted aqueous solubility (logS), MDCK cell permeability (MDCK), blood-brain partition coefficient ( $\log B B)$, percentage human oral absorption rate, etc. were analyzed.

\section{Results and Discussion}

\subsection{Synthesis of $\mathbf{6 a}-\mathbf{j}$}

The synthetic pathway of the compounds (6) is illustrated in Scheme 1. N-benzylisatin (3) was prepared using our previously reported method [31] by heating same equivalent of isatin (1) and benzyl bromide (2) at $80^{\circ} \mathrm{C}$ in dimethylformamide (DMF) for $12 \mathrm{~h}$. $\mathrm{N}$-benzylisatin monohydrazone (4) also was prepared using previously reported hydrazone formation method [27,32,33,37] using 1.2 equivalent of hydrazine hydrate and $\mathrm{N}$-benzylisatin (3) at refluxing condition for $3-4 \mathrm{~h}$ in alcoholic solution $(\mathrm{MeOH} / \mathrm{EtOH})$. Designed $N$-benzylisatin-aryl hydrazones $(\mathbf{6 a}-\mathbf{j})$ were synthesized by reacting compound 4 with substituted aryl aldehydes $(\mathbf{5 a}-\mathbf{j})$ in absolute ethanol in presence of catalytic amount of glacial acetic acid [27]. The choice of the substituents and its positions on the aromatic ring of benzaldehyde is to offer various electron withdrawal and donation on the aromatic ring which will, in turn, give different degrees of electron density on the biologically active parts of isatin molecule.

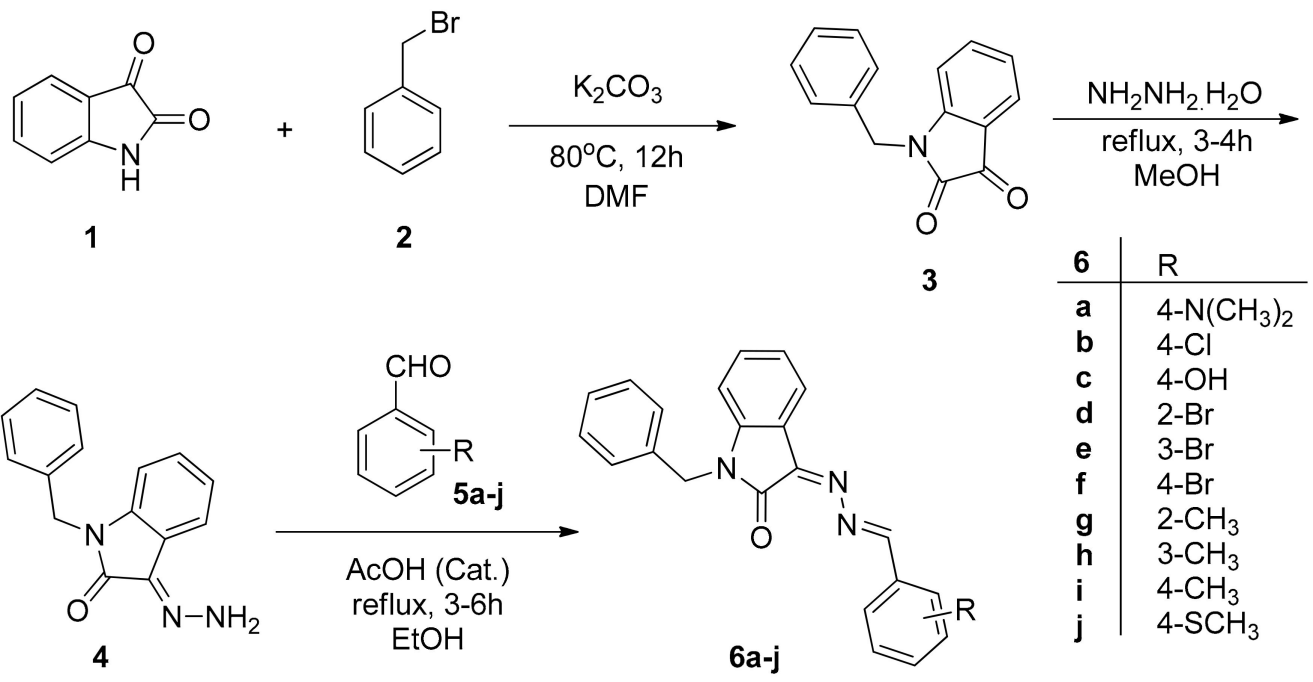

Scheme 1. Synthesis of $N$-benzylisatin-aryl hydrazones $(\mathbf{6 a}-\mathbf{j})$. 


\subsection{Biological Evaluation}

\subsubsection{Antimicrobial Evaluation of $\mathbf{6 a}-\mathbf{j}$}

Synthesized $N$-benzylisatin-aryl hydrazones $(\mathbf{6} \mathbf{a}-\mathbf{j})$ were evaluated for their antibacterial activity against two Gram-positive Staphylococcus aureus ATCC 29,213 and Bacillus subtilis ATCC 3366, four Gram-negative, Escherichia coli ATCC 25922, Klebsiella pneumoniae ATCC 13883, Salmonella typhi ATCC 25,566 and Pseudomonas aeruginosa ATCC 27,853 bacterial strains. As expected, the result of the antibacterial evaluation revealed no effect of these compounds $(\mathbf{6 a}-\mathbf{j})$ on selected strains of bacteria in concentration up to $100 \mu \mathrm{g} /$ disc (where no significant inhibition zones were detected). In case of antifungal activity, no inhibition zone was detected against Candida albicans NRRL Y-477. Excitingly, our hypothesis matched with the antimicrobial data of the evaluated compounds $\mathbf{6 a}-\mathbf{j}$, which are all having low molecular weight (353-418 Da), conforming to O'Shea and Moser's findings. In addition, the antimicrobial data of $\mathbf{6 a - j}$ also meet the rule of five (RO5) with $\log P$ value of 9.36-11.8 (please see Section 3.4), which is far more than to have anti-microbial activity. In case of antifungal activity, there were no inhibition zones detected against Candida albicans NRRL Y-477 either.

\subsubsection{Antiproliferative Activity of $\mathbf{6 a}-\mathbf{j}$}

The antiproliferative activity of compounds $\mathbf{6 a}-\mathbf{j}$ are listed in Table 1 as values of $\mathrm{IC}_{50}$. All the tested compounds displayed excellent antiproliferative activity against non-small cell lung cancer cell lines 'A549' and human cervical cancer cells lines 'HeLa' tested. Among them, compound 6c displayed the highest antiproliferative activity against the cell lines 'A549' $(4.35 \mu \mathrm{M})$ and 'Hela' $(4.09$ $\mu \mathrm{M})$. Whereas positive control gefitinib showed less antiproliferative activity against the cell lines 'A549' $(15.23 \mu \mathrm{M})$ and 'Hela' $(7.35 \mu \mathrm{M})$. Besides compound $6 \mathbf{c}$, another three bromo derivatives of $\mathrm{N}$-benzylisatin-aryl hydrazones $(\mathbf{6 d}, \mathbf{6 f}$ and $\mathbf{6 g}$ ) showed excellent antiproliferative activity, which is comparable to the control gefitinib. Detail explanations and SARs study are given below.

Table 1. Antiproliferative activity of $N$-benzylisatin-aryl hydrazones $(\mathbf{6 a}-\mathbf{j})$.

\begin{tabular}{|c|c|c|c|}
\hline \multirow{2}{*}{ Compound } & \multirow{2}{*}{$\begin{array}{l}C_{O}^{N}=N_{N}^{N} \\
(R=)\end{array}$} & \multicolumn{2}{|c|}{$\mathrm{IC}_{50}(\mu \mathrm{M})$} \\
\hline & & A549 & Hela \\
\hline 4 & - & $14 \pm 2.15$ & $46 \pm 1.30$ \\
\hline $6 a$ & $4-\mathrm{N}\left(\mathrm{CH}_{3}\right)_{2}$ & $23.7 \pm 0.69$ & $25.1 \pm 0.95$ \\
\hline $6 b$ & 4-Cl & $22 \pm 1.11$ & $14.8 \pm 0.94$ \\
\hline $6 c$ & $4-\mathrm{OH}$ & $4.35 \pm 0.05$ & $4 \pm 0.09$ \\
\hline $6 d$ & $2-\mathrm{Br}$ & $7.3 \pm 0.52$ & $6.7 \pm 0.17$ \\
\hline $6 e$ & $3-\mathrm{Br}$ & $41 \pm 1.20$ & $9.1 \pm 0.35$ \\
\hline $6 f$ & $4-\mathrm{Br}$ & $7.30 \pm 0.03$ & $7.4 \pm 0.20$ \\
\hline $6 g$ & $2-\mathrm{CH}_{3}$ & $7.5 \pm 0.11$ & $7.7 \pm 0.21$ \\
\hline $6 h$ & $3-\mathrm{CH}_{3}$ & $8.4 \pm 0.25$ & $13.4 \pm 0.35$ \\
\hline $6 i$ & $4-\mathrm{CH}_{3}$ & $17 \pm 0.70$ & $18.5 \pm 0.65$ \\
\hline $6 \mathbf{j}$ & $4-\mathrm{SCH}_{3}$ & $14.6 \pm 0.53$ & $9 \pm 0.86$ \\
\hline Gefitinib & - & $15.2 \pm 0.48$ & $7.3 \pm 0.42$ \\
\hline
\end{tabular}

Data represent means \pm SD obtained from three different experiments. Cell lines used as follows, non-small cell lung cancer cell lines 'A549' and human cervical cancer cells lines 'HeLa'. Gefitinib was used as positive controls. 


\subsection{Structure Activity Relationships (SARs) of $\mathbf{6} \boldsymbol{a}-\mathbf{j}$}

The compound, namely (E)-1-benzyl-3-hydrazonoindolin-2-one (4) showed moderate antiproliferative activity $(14.15 \mu \mathrm{M})$ against the non-small cell lung cancer cell lines 'A549' similar to that of gefitinib $(15.23 \mu \mathrm{M})$, but six-fold less active against human cervical cancer cells lines 'HeLa' $(46.03 \mu \mathrm{M})$. Compound 4 reacted with 4-substituted aryl aldehydes forming compound $6 \mathbf{a}$ having 4-(N,N-)-dimethyl group (6a), chloro group (6b) and methyl group (6i) decreased the antiproliferative activity than the gefitinib against both cell lines tested. While substituted by methylthio group (6j) shows similar antiproliferative activity comparing gefitinib. Interestingly, as depicted in Table 2, entry 6c, substituted by hydroxy group at 4-position showed higher antiproliferative activity which is around four fold than the gefitinib against non-small cell lung cancer cell lines 'A549' (4.35 $\mu \mathrm{M})$ and two fold higher than the HeLa cell lines $(4.09 \mu \mathrm{M})$. In case of bromo substituents at the 2- and 4-positions of the aryl ring, the compounds $\mathbf{6} \mathbf{d}$ and $\mathbf{6 f}$, respectively, the antiproliferative activity dramatically increased more than two-fold for A549, and similar for HeLa cell lines in comparison to the gefitinib. While addition at the 3-position by a bromo group (6e) and by a methyl group (6h) of the aryl ring showed similar or less antiproliferative activity comparing to gefitinib. Interestingly, the methyl group at 2-position (6g) increased the activity by two-fold compared to gefitinib, against A549 and similarly against HeLa cell lines. In conclusion, ortho and para substitution by bromo and hydroxy group proved to be the most active potential agents for non-small cell lung cancer cell lines 'A549' among the compound tested.

Table 2. Analysis of drug likeness properties and pharmacokinetic properties by QikProp for 6a-j.

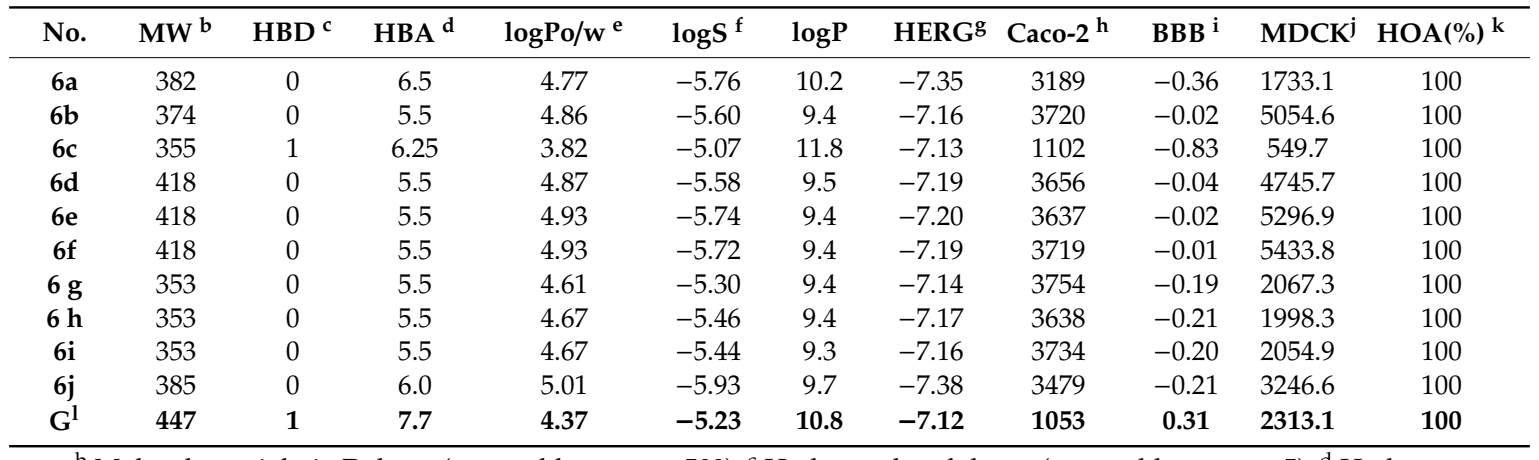

${ }^{\mathrm{b}}$ Molecular weight in Daltons (acceptable range: $<500$ ); ${ }^{\mathrm{c}}$ Hydrogen bond donor (acceptable range: $\leq 5$ ); ${ }^{\mathrm{d}}$ Hydrogen bond acceptor (acceptable range: $\leq 10$ ); ${ }^{\mathbf{e}}$ Predicted octanol/water partition coefficient (acceptable range: $-2-6.5$ ); ${ }^{\mathrm{f}}$ Predicted aqueous solubility, $\mathrm{S}$ in $\mathrm{mol} / \mathrm{dm}^{-3}$ (acceptable range: $-6.5-0.5$ ); g Predicted $\mathrm{IC}_{50}$ value for blockage of hERG K+ channels (concern: below -5$){ }^{\text {h }}$ Caco-2 value, permeability to Caco-2 (human colorectal carcinoma) cells in vitro; ${ }^{\text {I }}$ Blood-brain barrier permeability (acceptable range: -0.4); j Predicted apparent MDCK cell permeability in $\mathrm{nm} / \mathrm{sec}, \mathrm{QPPMDCK}=>500$ is great, $<25$ is poor; $\mathrm{k}$ Predicted human oral absorption on $0 \%$ to $100 \%$ scale $(<25 \%$ is poor and $>80 \%$ is high); $1 \mathrm{G}=$ Gefitinib.

\subsection{In Silico Drug Likeness Property Analysis}

In modern drug discovery approaches, evaluation of absorption, distribution, metabolism and excretion (ADME) of drug candidates impose significant value to the rational drug design. In vitro and in vivo ADME prediction has now become faster and more accurate with the computational chemistry tools which has been developed recently and aids the pharmaceutical industries to screen many compounds within a very short time [38]. In this experiment, we analyzed the predicted ADME values of the designed compounds (6a-j) and are summarized in Table 2. Since high molecular weight compounds are always less effective in terms of intestinal absorption, our synthesized compounds' $(\mathbf{6 a}-\mathbf{j})$ molecular weight (353-418Da) of this study are less than the established drug gefitinib (447Da), which supports the established parameters [9,39]. Subsequently, the studied compounds showed recommended values for hydrogen bond donor $(<5)$ and acceptor $(<10)$. Gefitinib showed HBD value of 1 and HBA value of 7.7 whereas the studied compounds showed HBD values of 0 (except $6 \mathbf{c}$ having HBD 1) and HBA values of maximum 6.5 (compound 6a), 6.25 (compound 6c), 6.0 (compound $\mathbf{6 j}$ ) 
and other compounds $\mathbf{6} \mathbf{b}, \mathbf{6} \mathbf{d}-\mathbf{6} \mathbf{i}$ having HBA values of 5.5 , which indicates superior values to the gefitinib (HBA values is 7.7). In 2002, Jorgensen and Duffy established a parameter to check the bioavailability of the drug compound which can be determined by octanol/water partition coefficient and solubility scoring where the recommended values are $-2-6.5$ and $-6.5-0.5 \mathrm{~mol} / \mathrm{dm}^{-3}$, respectively [40]. Compounds of this study showed scoring within the reference values including gefitinib. Octanol/water partition coefficient and solubility score in between 3.82-5.01 and -5.93--5.07, respectively. The hERG $\mathrm{K}+$ channel blockers are potentially toxic for the heart and thus the recommended range for predicted $\log \mathrm{IC}_{50}$ values for blockage of hERG K+ channels (loghERG) is $>-5$ [41]. Intriguingly, all the compounds of this study showed higher value for loghERG score $(>-7.13)$ than gefitinib $(-7.12)$ which proved their less toxicity than gefitinib. The Caco- 2 cell, considered as the reliable in vitro models to estimate oral drug absorption and transdermal delivery [42], was high for all compounds except gefitinib and compound $\mathbf{6 c}$, which signifies the improved and well oral drug absorption and transdermal delivery efficiency of the studied compounds than gefitinib. The blood-brain barrier separates CNS from blood and a successful compound must pass through the blood stream [43] which depends on several factors such as molecular weight which must be below 480 . Since our synthesized compounds have less molecular weight than the recommended values therefore its showed significant result. Madin-Darby canine kidney (MDCK) cell permeability is considered as the measurement of the blood-brain barrier permeability where greater than 500 is of great value and less than 25 indicates very poor result according to Jorgensen's rule of 3 [44]. Compounds $\mathbf{6 b}, \mathbf{6} \mathbf{d}, \mathbf{6 e}, \mathbf{6} \mathbf{f}$ and $\mathbf{6} \mathbf{j}$ gave much higher MDCK value than gefitinib while others showed around similar values except $\mathbf{6 c}$. The synthesized compounds also gave a predicted human oral absorption rate of $100 \%$. Taken together, all the designed compounds of this study showed higher predicted ADME values than gefitinib which can be tested further by in vitro and in vivo experiments to establish successful drug candidates.

\section{Conclusions}

A series of novel $\mathrm{N}$-benzylisatin-aryl-hydrazones were designed and synthesized with good to moderate yields for their antimicrobial and antiproliferative activity evaluation, for the development of potent anticancer therapeutics with no or minimal side effects. Six bacterial strains and a fungal strain were used for the antimicrobial screening of the synthesized compounds and no inhibitory effect was found on different microbes in concentration up to 100- $\mu \mathrm{g} / \mathrm{disc}$. On the other hand, four compounds showed two-to-four-fold antiproliferative activity than the FDA approved first-line treatments for lung cancer drug gefitinib. For example, $\mathrm{IC}_{50}$ of compound $\mathbf{6 c}$ is $4.35 \mu \mathrm{M}$, whereas gefitinib shows 15.23- $\mu \mathrm{M}$ concentration against non-small cell lung cancer cell lines 'A549.' In case of HeLa cell lines, antiproliferative activity of compound $\mathbf{6 c}$ also showed two-fold higher than the gefitinib. The strong inhibitory effect of $\mathbf{6 c}$, among the tested compounds, on the growth of cancerous cells accompanied by their complete safety on the growth of microbial cells, indicate a high level of target selectivity and a unique mechanism of action. Higher predicted ADME values were obtained than the known gefitinib. In our observation, this $\mathrm{N}$-benzylisatin-aryl-hydrazones can be a potential agent for anticancer therapeutics. Studies on the mechanism of action of antiproliferative activity of these derivative are ongoing and the results will be explored in due courses.

Supplementary Materials: The following are available online at http://www.mdpi.com/2076-3417/10/11/3669/s1, Figure of the ${ }^{13} \mathrm{CNMR}$ spectra of $\mathbf{6 a - j}$.

Author Contributions: Conceptualization, A.F.M.M.R. and H.S.A.-S.; data curation, H.A.A., I.S.I., A.Z.M., A.A. and M.A.; formal analysis, I.S.I., A.Z.M. and M.A.; funding acquisition, H.S.A.; investigation, H.S.A. and A.F.M.M.R.; methodology, H.A.A., I.S.I., A.Z.M., A.A. and M.A.; resources, H.S.A.-S. and A.F.M.M.R.; supervision, H.S.A.-S. and A.F.M.M.R; validation, H.S.A.-S.; writing—original draft, H.S.A.-S. and A.F.M.M.R.; writing一review and editing, H.S.A.-S. and A.F.M.M.R.; All authors have read and agreed to the published version of the manuscript.

Funding: This research was funded by the King Saud University, Research Center of the Female Campus for Scientific and Medical Studies. 
Acknowledgments: This research project was supported by a grant from the research center of the Female Campus for Scientific and Medical Studies, King Saud University, Riyadh, Saudi Arabia.

Conflicts of Interest: The authors declare no conflict of interest.

\section{References}

1. Crespo-Ortiz, M.P.; Wei, M.Q. Antitumor activity of artemisinin and its derivatives: From a well-known antimalarial agent to a potential anticancer drug. J. Biomed. Biotechnol. 2012. [CrossRef] [PubMed]

2. National Academies of Sciences, Engineering, and Medicine. The Drug Development Paradigm in Oncology: Proceedings of a Workshop; The National Academies Press: Washington, DC, USA, 2018. [CrossRef]

3. Burotto, M.; Manasanch, E.E.; Wilkerson, J.; Fojo, T. Gefitinib and erlotinib in metastatic non-small cell lung cancer: A meta-analysis of toxicity and efficacy of randomized clinical trials. Oncologist 2015, 20, 400-410. [CrossRef] [PubMed]

4. Hsiue, E.H.-C.; Lee, J.-H.; Lin, C.-C.; Yang, J.C.-H. Safety of gefitinib in non-small cell lung cancer treatment. Expert Opin. Drug Saf. 2016, 15, 993-1000. [CrossRef] [PubMed]

5. Ding, P.N.; Lord, S.J.; Gebski, V.; Links, M.; Bray, V.; Gralla, R.J.; Yang, J.C.-H.; Lee, C.K. Risk of treatment-related toxicities from EGFR tyrosine kinase inhibitors: A meta-analysis of clinical trials of gefitinib, erlotinib, and afatinib in advanced EGFR-mutated non-small cell lung cancer. J. Thorac. Oncol. 2016, 12, 633-643. [CrossRef] [PubMed]

6. Katakami, N.; Atagi, S.; Goto, K.; Hida, T.; Horai, T.; Inoue, A.; Ichinose, Y.; Koboyashi, K.; Takeda, K.; Kiura, K.; et al. LUX-Lung 4: A phase II trial of afatinib in patients with advanced non-small-cell lung cancer who progressed during prior treatment with erlotinib, gefitinib, or both. J. Clin. Oncol. 2013, 31, 3335-3341. [CrossRef]

7. Roy, S.; Hagen, K.D.; Maheswari, P.U.; Lutz, M.; Spek, A.L.; Reedijk, J.; van Wezel, G.P. Phenanthroline derivatives with improved selectivity as DNA-targeting anticancer or antimicrobial drugs. ChemMedChem 2008, 3, 1427-1434. [CrossRef]

8. O'Shea, R.; Moser, H.E. Physicochemical properties of antibacterial compounds: Implications for drug discovery. J. Med. Chem. 2008, 51, 2871-2878. [CrossRef]

9. Lipinski, C.A.; Lombardo, F.; Dominy, B.W.; Feeney, P.J. Experimental and computational approaches to estimate solubility and permeability in drug discovery and development settings. Adv. Drug Deliv. Rev. 2001, 46, 3-26. [CrossRef]

10. Vine, K.L.; Matesic, L.; Locke, J.M.; Skropeta, D. Recent highlights in the development of isatin-based anticancer agents. Adv. Anticancer Agents Med. Chem. 2013, 2, 254-312. [CrossRef]

11. Hajare, R.; Kulkarni, S.; Thakar, M.; Paranjape, R. Isatin anti-HIV agent: A review. World J. Pharm. Pharm. Sci. 2016, 5, 569-575. [CrossRef]

12. Pakravan, P.; Kashanian, S.; Khodaei, M.M.; Harding, F.J. Biochemical and pharmacological characterization of isatin and its derivatives: From structure to activity. Pharmacol. Rep. 2013, 65, 313-335. [CrossRef]

13. Li, C.; Zhang, F. Single step incorporation of isatin to enaminone: A recyclable catalyst towards assembly of diverse four ring fused pyrrolo[2,3,4-kl]acridin-1-ones. RSC Adv. 2016, 6, 75359-75364. [CrossRef]

14. Loloiu, G.; Loloiu, T.; Maior, O. Chemistry of isatin. Synthesis of 2,3-oxo-1H-pyrrolo[2,3-b]dibenzo-p-dioxins. Rev. Chim. (Buchar.) 1998, 49, 861-864.

15. Popp, F.D. Chemistry of isatin. Adv. Heterocycl. Chem. 1975, 18, 1-58.

16. Rajopadhye, M. The chemistry of isatin and isatin derivatives: Synthesis of novel ring systems. Diss. Abstr. Int. B 1988, 48, 2325-2326.

17. Sumpter, W.C. The chemistry of isatin. Chem. Rev. 1944, 34, 393-434. [CrossRef]

18. Da Silva, J.F.M.; Garden, S.J.; Pinto, A.C. The chemistry of isatins: A review from 1975 to 1999. J. Braz. Chem. Soc. 2001, 12, 273-324. [CrossRef]

19. Lashgari, N.; Mohammadi Ziarani, G. Synthesis of Heterocyclic Compounds Based on Isatin Through 1,3-Dipolar Cycloaddition Reactionsp; ARKIVOC: Gainesville, FL, USA, 2012.

20. Medvedev, A.; Igosheva, N.; Crumeyrolle-Arias, M.; Glover, V. Isatin: Role in stress and anxiety. Stress 2005, 8, 175-183. [CrossRef] 
21. Vine, K.L.; Matesic, L.; Locke, J.M.; Ranson, M.; Skropeta, D. Cytotoxic and anticancer activities of isatin and its derivatives: A comprehensive review from 2000-2008. Anti-Cancer Agents Med. Chem. 2009, 9, 397-414. [CrossRef]

22. Matesic, L.; Locke, J.M.; Bremner, J.B.; Pyne, S.G.; Skropeta, D.; Ranson, M.; Vine, K.L. N-Phenethyl and $\mathrm{N}$-naphthylmethyl isatins and analogues as in vitro cytotoxic agents. Bioorg. Med. Chem. 2008, 16, 3118-3124. [CrossRef]

23. Han, K.; Zhou, Y.; Liu, F.; Guo, Q.; Wang, P.; Yang, Y.; Song, B.; Liu, W.; Yao, Q.; Teng, Y.; et al. Design, synthesis and in vitro cytotoxicity evaluation of 5-(2-carboxyethenyl)isatin derivatives as anticancer agents. Bioorg. Med. Chem. Lett. 2014, 24, 591-594. [CrossRef] [PubMed]

24. Ibrahim, H.S.; Abou-seri, S.M.; Ismail, N.S.M.; Elaasser, M.M.; Aly, M.H.; Abdel-Aziz, H.A. Bis-isatin hydrazones with novel linkers: Synthesis and biological evaluation as cytotoxic agents. Eur. J. Med. Chem. 2016, 108, 415-422. [CrossRef]

25. Pervez, H.; Ahmad, M.; Zaib, S.; Yaqub, M.; Naseer, M.M.; Iqbal, J. Synthesis, cytotoxic and urease inhibitory activities of some novel isatin-derived bis-Schiff bases and their copper(II) complexes. MedChemComm 2016, 7, 914-923. [CrossRef]

26. Ganguly, S.; Debnath, B. Molecular docking studies and ADME prediction of novel isatin analogs with potent anti-EGFR activity. Med. Chem. (Los Angeles, CA, USA) 2014, 4, 558-568. [CrossRef]

27. Rahman, A.F.M.M.; Park, S.-E.; Kadi, A.A.; Kwon, Y. Fluorescein hydrazones as novel nonintercalative topoisomerase catalytic inhibitors with low DNA toxicity. J. Med. Chem. 2014, 57, 9139-9151. [CrossRef]

28. Dweedar, H.E.; Mahrous, H.; Ibrahim, H.S.; Abdel-Aziz, H.A. Analogue-based design, synthesis and biological evaluation of 3-substituted-(methylenehydrazono)indolin-2-ones as anticancer agents. Eur. J. Med. Chem. 2014, 78, 275-280. [CrossRef] [PubMed]

29. Eldehna, W.M.; Altoukhy, A.; Mahrous, H.; Abdel-Aziz, H.A. Design, synthesis and QSAR study of certain isatin-pyridine hybrids as potential anti-proliferative agents. Eur. J. Med. Chem. 2015, 90, 684-694. [CrossRef]

30. Islam, M.S.; Al-Majid, A.M.; El-Senduny, F.F.; Badria, F.A.; Rahman, A.F.M.M.; Barakat, A.; Elshaier, Y.A.M.M. Synthesis, anticancer activity, and molecular modeling of new halogenated spiro[pyrrolidine-thiazolo-oxindoles] derivatives. Appl. Sci. 2020, 10, 2170. [CrossRef]

31. Kadi, A.A.; Al-Shakliah, N.S.; Rahman, A.F.M.M. Synthesis and fragmentation behavior study of n-alkyl/benzyl Isatin derivatives present in small/complex molecules: Precursor for the preparation of biological active heterocycles. Mass Spectrom. Lett. 2015, 6, 65-70. [CrossRef]

32. Coffey, K.E.; Moreira, R.; Abbas, F.Z.; Murphy, G.K. Synthesis of 3,3-dichloroindolin-2-ones from isatin-3-hydrazones and (dichloroiodo)benzene. Org. Biomol. Chem. 2015, 13, 682-685. [CrossRef]

33. Popp, F.D. Potential anticonvulsants. IX. Some isatin hydrazones and related compounds. J. Heterocycl. Chem. 1984, 21, 1641-1645. [CrossRef]

34. Bauer, A.W.; Kirby, W.M.; Sherris, J.C.; Turck, M. Antibiotic susceptibility testing by a standardized single disk method. Am. J. Clin. Pathol. 1966, 45, 493-496. [CrossRef]

35. Stewart, M.J.; Watson, I.D. Standard units for expressing drug concentrations in biological fluids. Br. J. Clin. Pharmacol. 1983, 16, 3-7. [CrossRef] [PubMed]

36. Arifuzzaman, M.; Mitra, S.; Jahan, S.I.; Jakaria, M.; Abeda, T.; Absar, N.; Dash, R. A Computational workflow for the identification of the potent inhibitor of type II secretion system traffic ATPase of Pseudomonas aeruginosa. Comput. Biol. Chem. 2018, 76, 191-201. [CrossRef] [PubMed]

37. Murukan, B.; Kumari, B.S.; Mohanan, K. Synthesis, spectral, electrochemical and antibacterial studies of copper(II) complexes with isatin derived bishydrazone and different co-ligands. J. Coord. Chem. 2007, 60, 1607-1617. [CrossRef]

38. Ekins, S.; Waller, C.L.; Swaan, P.W.; Cruciani, G.; Wrighton, S.A.; Wikel, J.H. Progress in predicting human ADME parameters in silico. J. Pharmacol. Toxicol. Methods 2000, 44, 251-272. [CrossRef]

39. Ghose, A.K.; Viswanadhan, V.N.; Wendoloski, J.J. A knowledge-based approach in designing combinatorial or medicinal chemistry libraries for drug discovery. 1. A qualitative and quantitative characterization of known drug databases. J. Comb. Chem. 1999, 1, 55-68. [CrossRef]

40. Jorgensen, W.L.; Duffy, E.M. Prediction of drug solubility from structure. Adv. Drug Deliv. Rev. 2002, 54, 355-366. [CrossRef]

41. Chemi, G.; Gemma, S.; Campiani, G.; Brogi, S.; Butini, S.; Brindisi, M. Computational tool for fast in silico evaluation of hERG K+ channel affinity. Front. Chem. 2017, 5. [CrossRef] [PubMed] 
42. Kulkarni, A.; Han, Y.; Hopfinger, A.J. Predicting Caco-2 cell permeation coefficients of organic molecules using membrane-interaction QSAR. J. Chem. Inf. Comput. Sci. 2002, 42, 331-342. [CrossRef]

43. Clark, D.E. In silico prediction of blood-brain barrier permeation. Drug Discov. Today 2003, 8, 927-933. [CrossRef]

44. Lionta, E.; Spyrou, G.; Vassilatis, D.K.; Cournia, Z. Structure-based virtual screening for drug discovery: Principles, applications and recent advances. Curr. Top. Med. Chem. 2014, 14, 1923-1938. [CrossRef] [PubMed]

(C) 2020 by the authors. Licensee MDPI, Basel, Switzerland. This article is an open access article distributed under the terms and conditions of the Creative Commons Attribution (CC BY) license (http://creativecommons.org/licenses/by/4.0/). 